\title{
EXCITATION AND DAMPING OF P-MODES
}

\author{
ÅKE NORDLUND \\ Theoretical Astrophysics Center, and \\ Astronomical Observatory / NBIfAFG, \\ Juliane Maries Vej 30, 2100 Copenhagen Ø, Denmark \\ AND \\ ROBERT F. STEIN \\ Dept. of Physics and Astronomy, Michigan State University, \\ East Lansing, MI 48823, U.S.A.
}

\section{Introduction}

Millions of seismic eigenmodes are excited to low amplitudes in the Sun, with an amplitude spectrum that grows approximately as a power of the frequency until it reaches a maximum at $3 \mathrm{mHz}$, and then drops off again towards higher frequencies (Libbrecht, 1988; Libbrecht \& Woodard, 1991).

We would like to answer the following fundamental questions about these 'solar five-minute oscillations':

1. What excites the oscillations?

2. What damps the oscillations?

3. Why is the dominant period 5 minutes?

These questions are notoriously difficult to attack with analytical methods, because the properties of the oscillations depend critically on the properties of turbulent convection in the near-solar-surface layers. Even before the detailed mode structure was observed (Deubner, 1975), it was suspected that waves would be excited by the turbulent convection (Biermann, 1946; Biermann, 1948; Schwarzschild, 1948; Schatzman, 1949; Unno, 1964; Stein, 1967; Stein, 1968), and oscillations of the solar surface layers had been observed (Leighton et al., 1964; Evans \& Michard, 1962). Following the realization that a rich spectrum of global modes exist on the Sun (Ulrich, 1970; Leibacher \& Stein, 1971; Deubner, 1975; Ulrich \& Rhodes, 1977), a large number of attempts have been made to explain their excitation and damping properties (e.g., Ando \& Osaki, 1977; Goldreich \& Keeley, 1977; Gough, 1980; Antia et al., 1982; ChristensenDalsgaard \& Frandsen, 1983; Antia et al., 1987; Goldreich \& Kumar, 1988; Goldreich \& Kumar, 1990; Balmforth, 1992a; Balmforth, 1992c; Goldreich et al., 1994). The outcome of these studies for a long while actually confused the issue, because even the question of the stability of the modes could not be decided with any certainty by analytical means. One of the most impressive and laborious studies is the one by 
Balmforth (1992abc), who among many other results showed that a crucial factor for determining the stability of (in particular) the low-frequency modes is the response of the Reynolds stress to the presence of coherent modes. Balmforth showed that a commonly used approximation of these effects in terms of a turbulent viscosity significantly underestimates the stabilizing influence of convection, and that this explained the tendency to find overstability in works that adopted this approximation. He was able to fit the damping of low-frequency modes considerably better than in earlier works (possibly with the exception of Gough (1980)). Nevertheless, he concluded his series of papers with the statement "It would be wrong to rule out thermal overstability as the underlying cause of the five-minute oscillations on the basis of the current understanding".

By now, the consensus is that the modes are indeed stable and stochastically excited, but analytical predictions of their properties still contain major uncertainties. Thus, for example, Goldreich et al. (1994) used a number of (at least five) free parameters to fit the empirically determined stochastic excitation spectrum, openly admitting that some of the parameters had to be assigned unexpected values.

The main problem with analytical estimates of the excitation and damping is indeed that one cannot avoid the use of a number of free parameters, and that the results depend quite sensitively on the values adopted for some of these. Numerical simulations offer a way forward in this situation. The hydrodynamic equations that describe the solar surface layers contain no free parameters per se, and provided that sufficiently realistic physics is used, numerical solutions of these equations should provide increasingly accurate representations, as the numerical resolution is increased. The main problems with such an approach are, on the one hand that it is difficult to estimate the actual accuracy of the solutions, and on the other hand that, even with accurate numerical solutions it is non-trivial to extract the desired quantitative and qualitative information from the available data.

In what follows, we provide a brief overview of what numerical simulations can tell us about the excitation and damping of the solar five-minute oscillations. The discussion is based on numerical simulations of the solar surface layers, along the lines of previous work (Nordlund, 1982; Nordlund, 1985; Stein \& Nordlund, 1989; Nordlund \& Dravins, 1990; Nordlund \& Stein, 1990; Rast et al., 1993; Stein \& Nordlund, 1997). The numerical models include the relevant physics of the solar surface layers: ionization, radiative energy transfer, etc., and have been checked against a number of observational diagnostics. The results are found to be consistent with statistics of solar granulation (Wöhl \& Nordlund, 1985; Nordlund et al., 1997), spectral line widths and shapes (Dravins et al., 1981; Dravins et al., 1986; Dravins \& Nordlund, 1990a; Dravins \& Nordlund, 1990b; Spruit et al., 1990; Nordlund, 1991; Kiselman \& Nordlund, 1995), the depth of the solar convection zone (Nordlund \& Stein, 1997). and the p-mode frequency behavior (Rosenthal et al., 1998).

In previous work (Stein \& Nordlund, 1991) we have made use of long simulation runs with spontaneously excited modes to show, e.g., that the stochastic excitation due to entropy fluctuations significantly exceeds that due to the turbulent pressure (Reynolds stress) fluctuations. These experiments were 'passive', in the sense that the modes were left to establish the amplitudes that are appropriate for the sparse mode spectra of the relatively small boxes that are used in the numerical experiments.

To study specifically the excitation and damping of the oscillations, we have also performed a number of 'active experiments', where particular modes are initially either excessively excited or excessively damped. By actively exciting particular modes to 
high amplitude it becomes easier to study the mode damping, and by actively damping the modes it becomes easier to study the mode excitation.

To analyze excitation and damping mechanisms, it is advantageous to separate horizontal mean values and their coherent fluctuations (the modes), and refer to the remaining fluctuations as 'the turbulent convection' - of course with the understanding that correlations among the fluctuating variables also may exhibit a partly coherent behavior, and thus feed back into the behavior of the 'modes'. We summarize such a formalism in the next Section, and demonstrate the separation between 'modes' and 'convection'. In Section 3 we use the formalism to study and discuss the excitation mechanisms, and in Section 4 we treat the damping in a similar way. In the concluding section we summarize what we have thus learnt about the excitation and damping mechanisms.

\section{Formalism}

The formalism summarized here is an extension of the one used by Stein \& Nordlund (1991) and by Rosenthal et al. (1998). It is presented in more detail by Nordlund \& Stein (1998).

\subsection{1-D EQUATIONS}

For a one-dimensional envelope, the equations for radial near-surface motions may be written (in Lagrangian coordinates):

$$
\begin{aligned}
\frac{D}{D t}(\rho) & =-\rho \frac{\partial}{\partial z}\left(u_{z}\right) \\
\rho \frac{D}{D t}\left(u_{z}\right) & =-\frac{\partial}{\partial z}\left(P-\rho \sigma_{z z}\right)+\rho g \\
\rho \frac{D}{D t}\left(e+\frac{1}{2} u_{z}{ }^{2}\right) & =-\frac{\partial}{\partial z}\left(u_{z} P+F\right)+\rho u_{z} g
\end{aligned}
$$

where $u_{z} P$ is the acoustic flux and $F$ represents other fluxes.

In a true 1-D model, the only other fluxes are due to radiation and heat conduction. The turbulent convective flux that is often included in the energy equation of otherwise one-dimensional models cannot be determined without resorting to a multidimensional picture (if only for making order of magnitude or scaling estimates).

\subsection{3-D EQUATIONS}

The three-dimensional version of Eqs. (1)-(3) only differ by having additional, horizontal transport terms (but support an incomparably richer spectrum of motions):

$$
\begin{aligned}
\frac{D}{D t}(\rho) & =-\rho \frac{\partial}{\partial x_{j}}\left(u_{j}\right) \\
\rho \frac{D}{D t}\left(u_{i}\right) & =-\frac{\partial}{\partial x_{i}}(P)+\frac{\partial}{\partial x_{j}}\left(\rho \sigma_{i j}\right)+\rho \delta_{i z} g \\
\rho \frac{D}{D t}\left(e+\frac{1}{2} u^{2}\right) & =-\frac{\partial}{\partial x_{j}}\left(u_{j} P+F_{j}\right)+\rho u_{z} g
\end{aligned}
$$


There exist several interaction mechanisms between 'convection' and 'modes' (the quotes are there to emphasize that it is non-trivial to actually separate these effectshowever in what follows we generally drop the quotes). The convective energy flux carries almost all of the solar luminosity at depths larger than a few hundred kilometers below the optical surface, and the 'turbulent pressure' associated with convection is significant in a shallow layer near the solar surface. Since convection is a non-stationary process, there are significant, incoherent fluctuation in these transport processes. Such fluctuations necessarily give rise to random deviations from the average vertical balance of energy and vertical momentum, and hence, to sources of acoustic 'noise'. Conversely, in the presence of coherent wave-like perturbations around the average state, the convective transport of heat and momentum will be influenced (modulated), in general with some delay relative to the variations in the background state. Such coherent fluctuations of the average transport of energy and momentum in turn feed back onto the wave propagation; i.e., part of the coherent fluctuations of the convective medium are mediated by the convection itself.

\subsection{DENSITY-WEIGHTED AVERAGING}

In order to turn such qualitative remarks into quantitative expressions, it is helpful to use a consistent set of definitions for averages and fluctuations. Stein \& Nordlund (1991) adopted density-weighted averages for per-unit-mass variables (here denoted by lower case), and straight averages for per-volume quantities (here denoted by upper case).

$$
\begin{aligned}
\bar{F} & =\langle F\rangle_{x y} \\
\bar{f} & =\langle\rho f\rangle_{x y} /\langle\rho\rangle_{x y}
\end{aligned}
$$

where \langle\rangle stands for horizontal averages. With these definitions it follows that

$$
\begin{aligned}
\left\langle F^{\prime}\right\rangle \equiv\langle F-\bar{F}\rangle & =0, \\
\left\langle\rho f^{\prime}\right\rangle \equiv\langle\rho f-\bar{\rho} \bar{f}\rangle & =0, \\
\langle\rho f g\rangle & =\bar{\rho} \bar{f} \bar{g}+\left\langle\rho f^{\prime} g^{\prime}\right\rangle .
\end{aligned}
$$

The 'chain rule' (11) follows from Eqs. (9)-(10). Repeated use of the chain rule results in precise definitions for the total kinetic energy and kinetic energy flux, in terms of contributions from coherent motions and fluctuations:

$$
\begin{aligned}
\left\langle\frac{1}{2} \rho u^{2}\right\rangle & =\frac{1}{2} \bar{\rho} \bar{u}^{2}+\frac{1}{2}\left\langle\rho u^{\prime 2}\right\rangle=\bar{\rho} \bar{e}_{\mathrm{kin}, \text { mode }}+\bar{\rho} \bar{e}_{\mathrm{kin}, \text { turb }} \\
\left\langle\frac{1}{2} \rho u^{2} u_{z}\right\rangle & =\frac{1}{2} \bar{\rho} \bar{u}^{2} \bar{u}_{z}+\left\langle\frac{1}{2} \rho u^{\prime 2} u_{z}^{\prime}\right\rangle+\left\langle\rho u_{z}^{\prime 2}\right\rangle \bar{u}_{z}+\left\langle\frac{1}{2} \rho u^{\prime 2}\right\rangle \bar{u}_{z} \\
& =\bar{F}_{\text {kin,mode }}+\bar{F}_{\text {kin,turb }}+\bar{P}_{\text {turb }} \bar{u}_{z}+\bar{\rho} \bar{e}_{\text {kin,turb }} \bar{u}_{z}
\end{aligned}
$$

Equation (12) expresses the total kinetic energy as a sum of the kinetic energy of the mean motion and the kinetic energy of the convection. Equation (13) expresses the total kinetic energy flux as the sum of the kinetic energy flux of the mean, the turbulent kinetic energy flux, the acoustic fluxes associated with the horizontally averaged turbulent pressure and velocity, and the advection of average turbulent kinetic energy. 
Carrying on with a similar analysis of the other hydrodynamic equations, using the density-weighted averaging, one may derive a set of exact, horizontally averaged 3-D equations (Nordlund \& Stein, 1998).

\subsection{SEPARATION BETWEEN OSCILLATIONS AND CONVECTION}

For purely radial modes, we consider the fluctuations in time of the barred, horizontally averaged quantities as the variables belonging to the oscillations, and the primed 3 -D fluctuations as the ones associated with the convection. Qualitatively similar results are to be expected for low $\ell$ non-radial modes, since they are nearly radial in the surface layers. In fact, since numerical models of the solar surface region typically have horizontal sizes $\sim 10 \mathrm{Mm}$, the smallest non-zero $\ell$-values that could be studied would have $\ell$ in excess of 400 .

\section{Stochastic Excitation}

The kinetic energy of the mean obeys

$$
\bar{\rho} \frac{D}{D t}\left(\frac{1}{2} \bar{u}_{z}^{2}\right)=-\frac{\partial}{\partial z}\left(\bar{u}_{z} \bar{P}_{\text {gas }}+\bar{u}_{z} \bar{P}_{\text {turb }}\right)+\left(\bar{P}_{\text {gas }}+\bar{P}_{\text {turb }}\right) \frac{\partial \bar{u}_{z}}{\partial z}+g \bar{\rho} \bar{u}_{z} .
$$

Apart from boundary effects, the work comes from the term

$$
\left(\bar{P}_{\text {gas }}+\bar{P}_{\text {turb }}\right) \frac{\partial \bar{u}_{z}}{\partial z}=-\left(\bar{P}_{\text {gas }}+\bar{P}_{\text {turb }}\right) \frac{D}{D t}(\ln \bar{\rho})=-\bar{P}_{\text {tot }} \frac{D}{D t}(\ln \bar{\rho})
$$

Work arises from correlations in time between fluctuations in the two factors, $\delta \bar{P}_{\text {tot }}(t)$ and $\frac{D}{D t}(\ln \bar{\rho})$. The fluctuations consist of both coherent and incoherent contributions, where the coherent contributions include the in-phase (pressure relative to density) and out-of-phase response of the convection to the coherent $\mathrm{p}$-mode motions . The in-phase (adiabatic) part of the coherent response perturbs the frequency of the mode, while the out-of-phase (non-adiabatic) part causes damping (or driving) of the mode. The relation between coherent pressure and density perturbations may formally be written

$$
\delta \ln \bar{P}_{\text {tot }}=\gamma \delta \ln \bar{\rho}
$$

where $\gamma=\gamma(z, \omega)$ is a complex depth- and frequency-dependent factor. The function $\gamma(z, \omega)$ may be measured empirically in the numerical simulations, for example by performing "active" experiments, where a few modes are excited to significant amplitudes. The experiments may be "tuned", by varying the depth of the models, so that a suitable range of eigenfrequencies is covered.

The work done by the convection on the modes is the convolution of the out-ofphase, non-adiabatic, pressure fluctuations with the modal density fluctuations.

$$
W=-\int d t \int \frac{d m}{\bar{\rho}} \delta \bar{P}_{\text {tot }} \frac{D}{D t}(\ln \bar{\rho})=\int d t \int d z \delta \bar{P}_{\text {tot }} \frac{\partial \dot{\xi}}{\partial r}
$$

where $d m$ is a Lagrangian mass element, and $\xi$ is a Lagrangian displacement that satisfies

$$
\bar{u}_{z}=\frac{D}{D t}(\xi) \equiv \dot{\xi}
$$




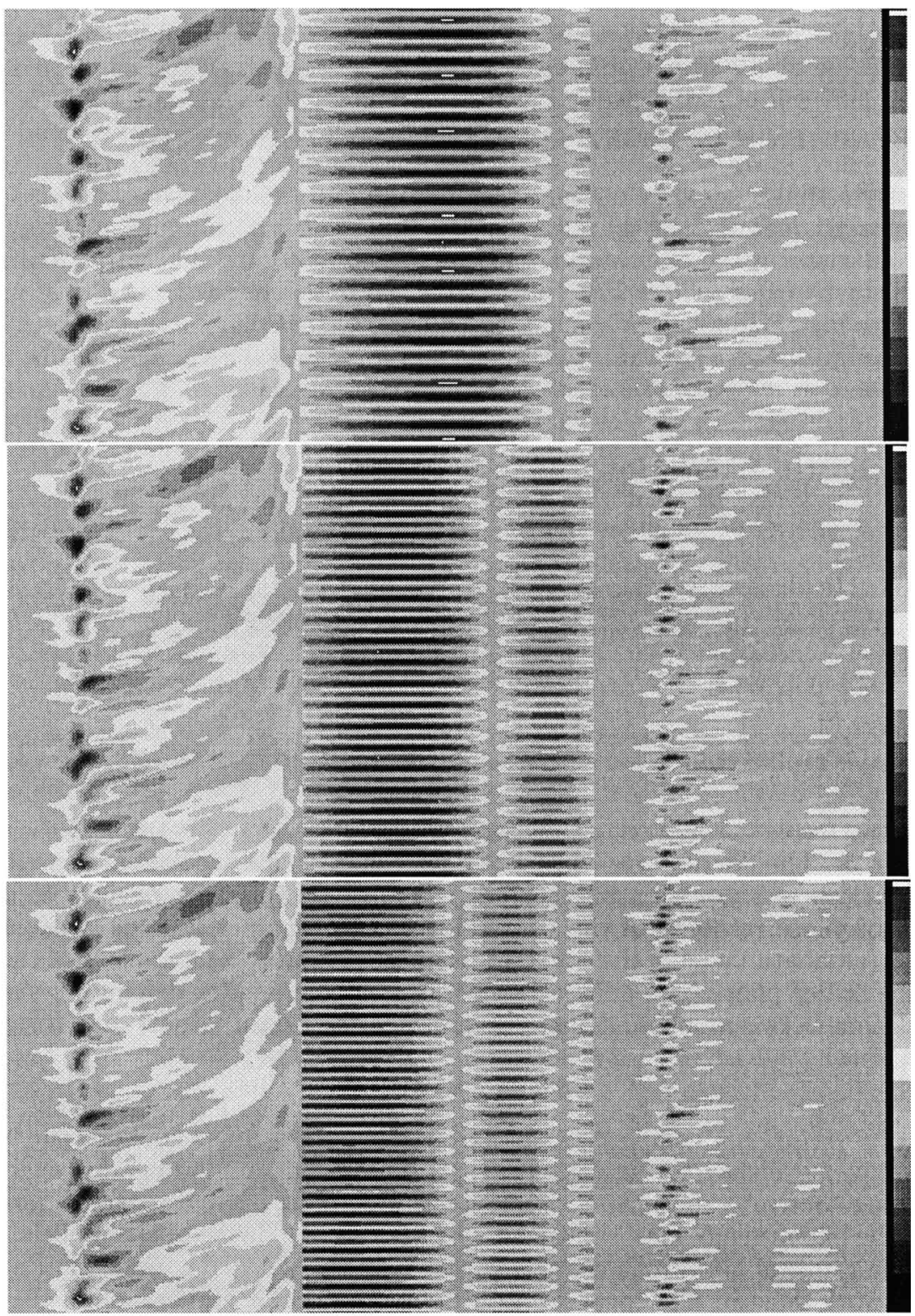

Figure 1. Images showing the integrand in the stochastic work integrals for radial modes at 3 , 4 , and $5 \mathrm{mHz}$. The leftmost part of each panel shows the horizontally averaged, non-adiabatic pressure fluctuation from an 80 minutes time sequence of a $6 \times 6 \times 3 \mathrm{Mm}$ numerical simulation with a spatial resolution of $125 \times 125 \times 82$ (Nordlund \& Stein, 1998). The middle part of each panel shows the mode pattern, and the rightmost part shows the product of the mode pattern and the convective fluctuations. (Color versions of this and other figures are available at URL http://www.astro.ku.dk/ aake/talks/Kyoto97.) 
Figure 1 shows the the horizontally averaged incoherent (convective) pressure fluctuations, the coherent (modal) density fluctuations and the product of the two, for three particular frequencies. High frequency modes tend to "chop up" the convective fluctuations in time, while low-frequency modes tend to sample more than one convective fluctuation per cycle. In addition, high- and low-frequency modes sample the convective perturbations differently in depth, with high-frequency modes tending to also chop up the convective fluctuations in the depth direction.

For a particular realization of the convection, the net result depends of course on the particular phase with which the modes are overlaid onto the convective fluctuations. Symbolically, we may write this as

$$
a_{i} \rightarrow a_{i}+\delta a_{i} e^{i\left(\phi-\phi_{0}\right)}
$$

where $a_{i}$ is the amplitude of a particular mode, and $\delta a_{i}$ is the perturbation of that amplitude when the mode is assigned the phase $\phi_{0}$. Averaging over phase one obtains a net contribution to the mode energy

$$
\left|a_{i}\right|^{2} \rightarrow\left|a_{i}\right|^{2}+\left|\delta a_{i}\right|^{2}
$$

A more detailed analysis (Nordlund \& Stein, 1998) results in the following expression for the average rate of stochastic excitation per unit surface area:

$$
\frac{\Delta\left\langle E_{\omega}\right\rangle_{\mathrm{ens}}}{\Delta t}=\frac{\left|\int_{r} d r \delta P_{\omega} \partial \xi_{\omega} / \partial r\right|^{2}}{\Delta \nu \int_{r} d r\left|\xi_{\omega}\right|^{2} \rho\left(\frac{r}{R}\right)^{2}}
$$

where $\delta P_{\omega}$ is the Fourier amplitude of the incoherent pressure fluctuations, and $\Delta \nu$ is the frequency resolution with which $\delta P_{\omega}$ is measured (note that in the limit $\Delta \nu \rightarrow 0$ the power per unit frequency interval $\left|\delta P_{\omega}\right|^{2} / \Delta \nu$ is independent of $\Delta \nu$ for a random function of time). Because the stochastic fluctuations are concentrated near the surface, the integral samples the mode compressibility $\partial \xi_{\omega} / \partial r$ in a shallow layer near the surface, where modes below about $3 \mathrm{mHz}$ are evanescent. The result is that there is an overall scaling with frequency that comes from the asymptotic behavior $\left(\partial \xi_{\omega} / \partial r \sim \omega^{2} \xi\right)$ of evanescent modes (Osaki, 1990; Balmforth, 1992c; Goldreich et al., 1994).

It is noteworthy that the only property of the turbulent convection that enters is the stochastic fluctuation of the total pressure. This is in contrast to the many approximate, analytic expressions for the stochastic excitation that have been derived (Lighthill, 1952; Unno, 1962; Moore \& Spiegel, 1964; Unno, 1964; Stein, 1967; Goldreich \& Keeley, 1977; Christensen-Dalsgaard \& Frandsen, 1983; Goldreich \& Kumar, 1988; Goldreich \& Kumar, 1990; Balmforth, 1992c; Goldreich et al., 1994). These expressions, one way or another, correspond to the exact expression with its fluctuations of the total pressure, but they have been expanded into a multitude of approximate terms, with possibly obscure cancellation effects. Balmforth (1992c) indeed pointed out that such expressions are non-unique, and that even the distinction between monopole, dipole and quadrupole source terms is ambiguous. Equation (21) short-circuits such ambiguities, by expressing the driving directly in terms of the one relevant quantity: the total pressure fluctuation, $\delta \bar{P}_{\text {tot }}=\delta P_{\text {gas }}+\delta P_{\text {turb }}$. Such an equation is of course only useful if one has access to numerical data that make it possible to evaluate the resulting expression. Fig. 2 shows the result of evaluating Eq. (21) from an 80 minute interval of numerical simulations (Nordlund \& Stein, 1998). 


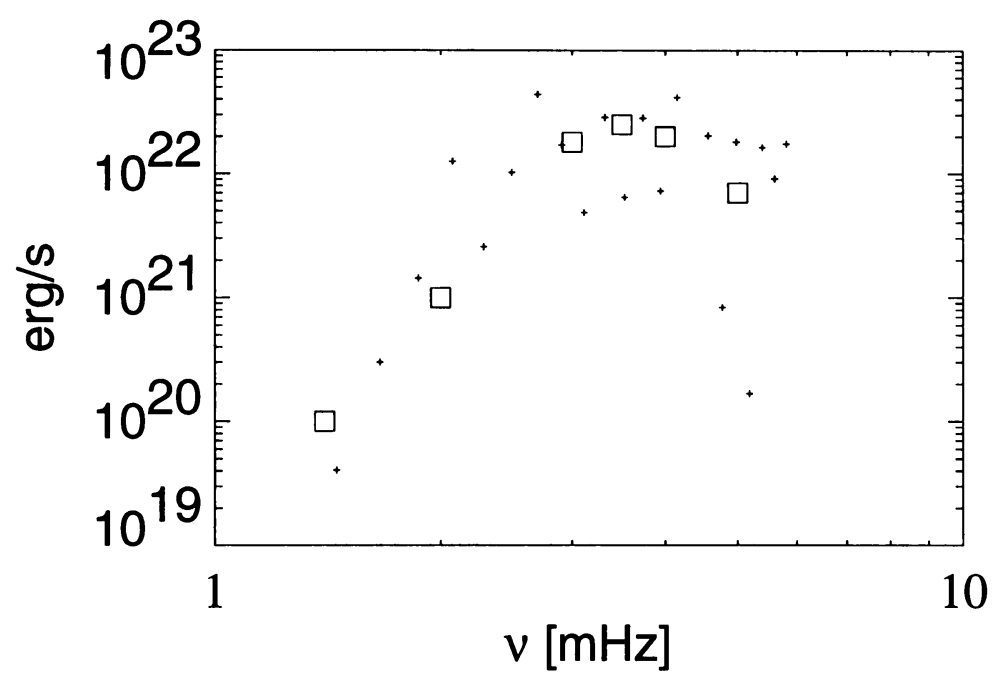

Figure 2. The stochastic energy input, scaled to the total solar area. The small plus symbols are from the numerical experiments, the large squares are determined from solar observations (cf. Fig. 7, Goldreich et al., 1994).

\subsection{EXCITATION EVENTS}

Using Eq. (21), and the direct visualizations of the work integral in Fig. 1, one may study not only the statistically averaged contributions to the stochastic driving, but also the contributions from particular events. Figure 3 shows the correlation between the instantaneous, horizontally averaged surface intensity and the contributions to the work integral from the surface layers. It is evident that there is a tight correlation, illustrating that it is indeed the fluctuating rate of cooling at the surface that is a main source of the stochastic excitation in the surface layers. Fig. 4 illustrates this further, by showing the contributions to the horizontal average over a short interval of time. The relatively large dynamic range of intensity fluctuations $(\sim 20 \% \mathrm{rms})$ makes it difficult to distinguish individual contributions to the average intensity fluctuation in the fully resolved images, but smearing the images to a resolution roughly corresponding to the size of granules helps. The horizontally averaged intensity fluctuation is most of the time the net effect of the changes in several granules, but occasionally, individual events dominate. The rapid drop in average intensity from the 3rd to the 5th panel in Fig. 4 is due mostly to the rapid cooling of a single granule fragment, resulting in a darkening that gradually disappears, as new granules develop in the area.

It is probably extreme events similar to this one that are the "seismic events" identified by Goode et al. (1992) (cf. Rimmele et al., 1995; Restaino et al., 1993). As shown by Goode et al. (1992), a sufficiently rapid cooling leads to an event characterized by a noticeable phase propagation, consisting of first an upward propagating expansion wave, followed by a ringing "wake". In the context of the current discussion, such events appear as "tips of the icebergs": they are the most extreme events in the 


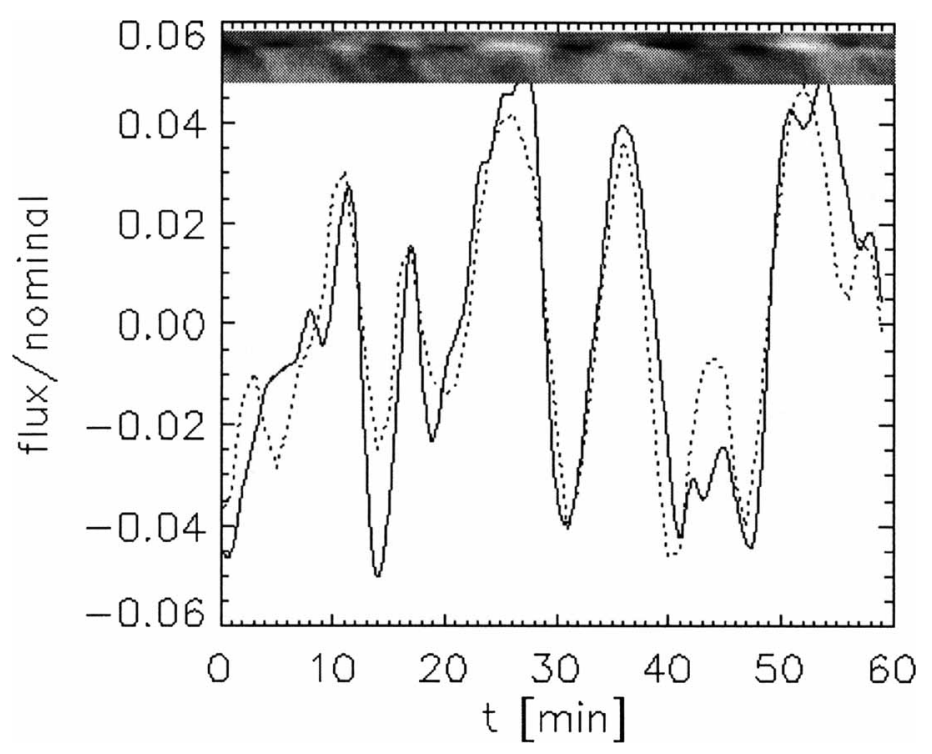

Figure 3. The average surface radiation intensity (dashed) and the non-adiabatic pressure fluctuation (full drawn, and image strip) in the surface layers of a numerical simulation.

ensemble of fluctuations that constitute the acoustic source. A detailed comparison between observations and synthetic spectral line data generated from the numerical simulations is necessary to estimate what fraction of the excitation that comes from identifiable seismic events.

\section{Damping}

Damping is a linear effect, that arises because of a systematic phase difference between the coherent fluctuations of pressure and density. The work done by the coherent motions is again proportional to the work integral, Eq. (17), where $\delta \bar{P}_{\text {tot }}$ is now the coherent, non-adiabatic part of the pressure fluctuations. The resulting expression for the damping rate is

$$
\frac{1}{E_{\omega}} \frac{d E_{\omega}}{d t}=\frac{2 \int_{r} d r \operatorname{Im}\left[\delta P_{\omega}\right] \partial \xi_{\omega} / \partial r}{\omega \int_{r} d r\left|\xi_{\omega}\right|^{2} \rho\left(\frac{r}{R}\right)^{2}}
$$

where $\operatorname{Im}\left[\delta P_{\omega}\right]$ is the imaginary part of the coherent fluctuation of the total pressure. As for the excitation, this integral can in principle be evaluated directly from numerical simulations. It is, however, non-trivial to separate the coherent part of the fluctuation from the stochastic ones in simulations where the damping and excitation are approximately in balance. One obtains a much "cleaner" situation by performing active experiments, where an initial "kick" excites a few oscillatory modes to a relatively high amplitude. By using higher amplitudes than the modes spontaneously attain, one obtains the benefit that the coherent fluctuations dominate over the stochastic ones, and that, consequently, the linear damping dominates over the 


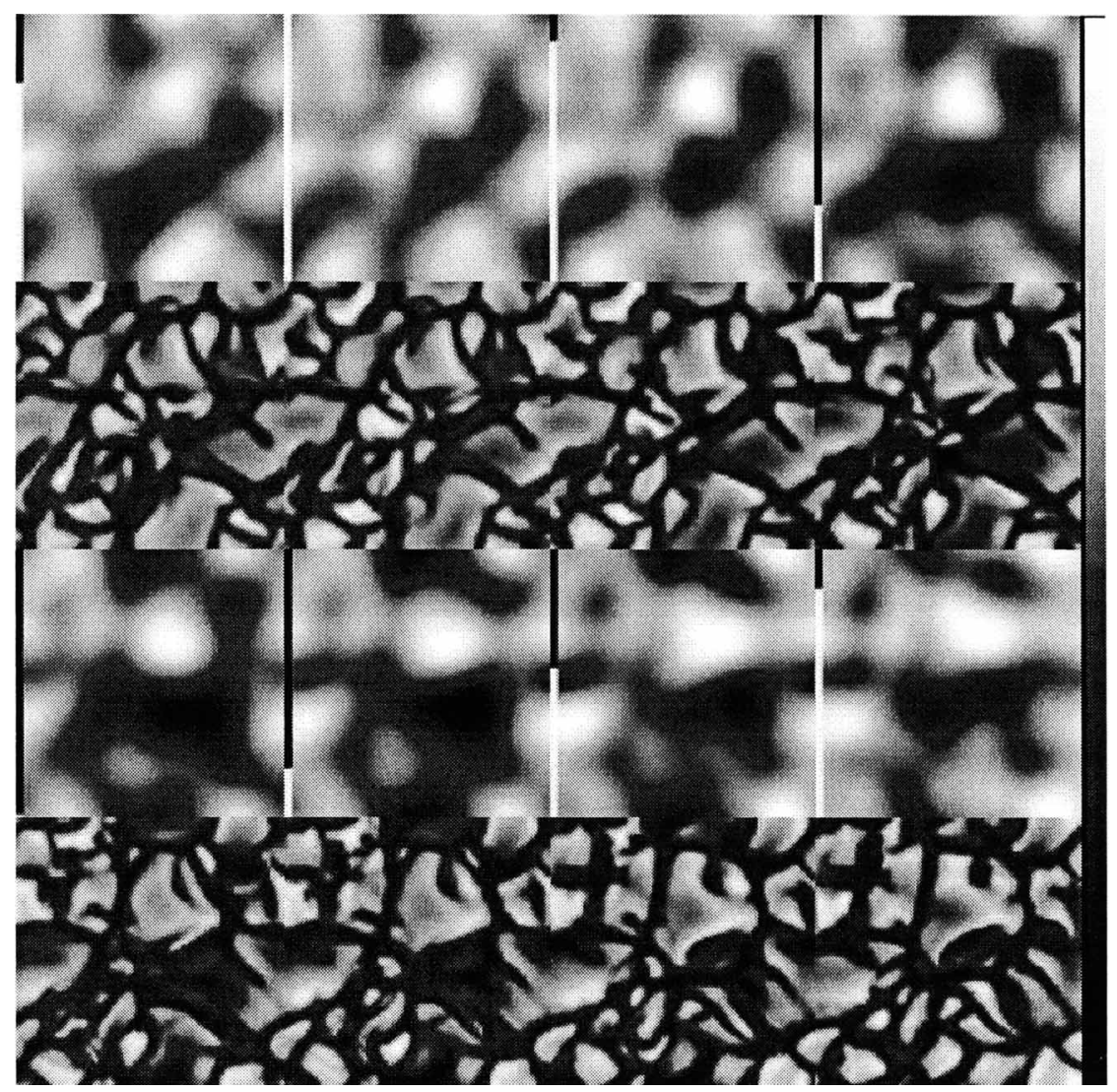

Figure 4. A time sequence of surface radiation intensity images at one minute time intervals, covering eight solar minutes. The upper rows of panels have been convolved with a Gaussian kernel, producing a spatial resolution comparable with the size of granules. The intensity contrast is thus reduced from $\sim 20 \%$ to a few percent, making it easier to locate the contributions to changes of the overall average intensity, which is indicated by the thin bars between the individual panels (the range is $\approx \pm 4 \%$ ).

stochastic excitation. In such situations, Eq. (22) may indeed be used to evaluate the damping rate directly from the numerical data.

With the current set of models, this works well for frequencies of the order of 3 $\mathrm{mHz}$; there, most of the contribution to the integral in Eq. (22) comes from layers close to the surface, and the damping rates are indeed consistent with the observed FWHM line widths $\sim 1 \mu \mathrm{Hz}$ near $3 \mathrm{mHz}$ (Libbrecht, 1988; Toutain \& Frölich, 1992; Bachmann et al., 1993). For lower frequencies, one encounters two (related) difficulties. First, the lowest eigenfrequencies of the current set of active experiments lies at 2.6 $\mathrm{mHz}$; i.e., it is not possible to measure the damping at lower frequencies from this set of experiments. One might consider performing continuously forced experiments, where an external volume force and appropriate boundary conditions maintain perturbations modeled after known eigenmodes, for those surface parts of the modes that are covered by the numerical simulations. Here one encounters the second difficulty, namely that 
the contribution to the work integral at low frequencies actually contains significant contributions from deeper layers. This may be appreciated by considering the variation with depth of the factors that enter into the integral in Eq. (22). The factor $\partial \xi / \partial r$ increases rapidly with depth for eigenmodes of low frequency. The overall magnitude of the turbulent pressure also increases with depth, even though the ratio $P_{\text {turb }} / P_{\text {gas }}$ peaks near the surface. Thus, unless the relative fluctuation of the turbulent pressure $\delta P_{\text {turb }}(\omega) / P_{\text {turb }}$ decreases rapidly with depth, one should expect that the work integral picks up significant contributions from deeper layers (cf. Fig. 14, Balmforth, 1992a). In order to account for these contributions, there seems to be no way around extending the models so that one can measure the damping using eigenmodes that are contained within the box.

Given that such measurements of the damping can be made, there is still the task of identifying the physical causes of the phase shift of the coherent fluctuations of the total pressure. The imaginary part of the gas pressure fluctuations is easily understood: it stems from the non-adiabatic terms in the energy equation. The factors that control the phase of the turbulent pressure fluctuations are less evident from first principles. However, using the technique of density-weighted averaging, one may derive a differential equation that describes the time evolution of the turbulent pressure (Nordlund \& Stein, 1998):

$$
\begin{aligned}
\frac{D}{D t}\left(P_{\text {turb }}\right)= & -\frac{\partial\left\langle\rho u_{z}^{\prime 2} u_{z}^{\prime}\right\rangle}{\partial z} & & \text { vertical Reynolds stress transport } \\
& +2\left\langle u_{z}^{\prime}\left(g \rho-\frac{\partial P_{\text {gas }}}{\partial z}\right)\right\rangle & & \text { buoyancy work } \\
& -3 P_{\text {turb }} \frac{\partial \bar{u}_{z}}{\partial z} & & \text { expansion / compression } \\
& +2\left\langle\rho u_{j}^{\prime} \sigma_{j z}\right\rangle, & & \text { viscous dissipation }
\end{aligned}
$$

where the terms have been labeled with their physical interpretation.

It is instructive to visualize the left and right hand side terms in this equation as two-dimensional (depth-time) images (Fig. 5). Examination reveals that the coherent response of the buoyancy work is particularly crucial. In an equilibrium situation, the buoyancy work is positive, and is balanced by the viscous dissipation and redistributed by the transport term. Below the surface, the buoyancy work comes predominantly from the core of the downdrafts, that have a small horizontal area filling factor, and hence large downward velocities and smaller than average gas pressure gradients, so are subject to net downwards forces. The response to a mode perturbation comes mainly from the modulation of the gas pressure gradient: in the upwards acceleration phase that precedes the expansion phase, the increased gas pressure gradient reduces or reverses the buoyancy work. The result is a tendency for the turbulent pressure to be 180 degrees out-of-phase with the density fluctuation.

When combined with the other terms in the equation for the turbulent pressure, the result is that the turbulent pressure fluctuations tend to be in phase with the density fluctuations at high frequencies, because of the compression term, tend to be in anti-phase with $\delta \bar{\rho}$ at low frequencies, and pass between the two extremes through phase shifts that lead to damping of the modes. 


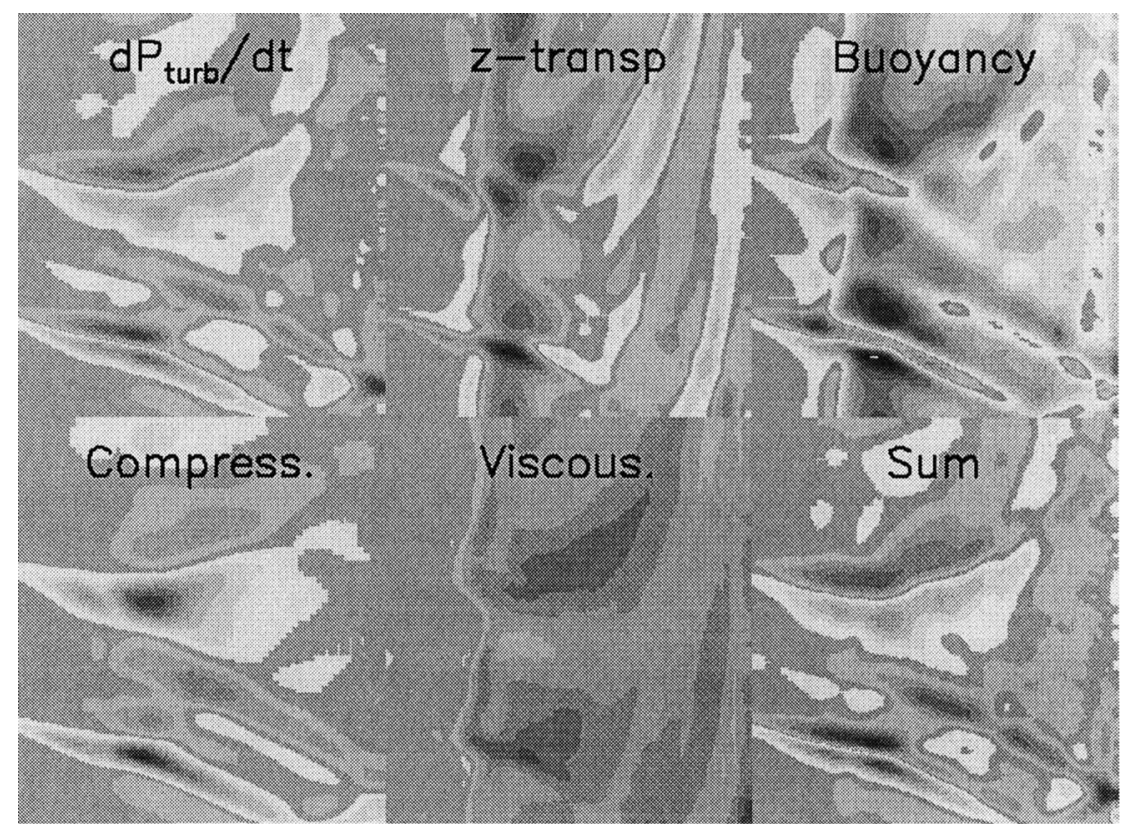

Figure 5. A visualization of the Eq. (23) left hand side, the right hand side terms, and their sum. In each panel, depth increases from left to right, and time increases from bottom to top.

\section{Conclusions}

The results of numerical simulations are helpful in several ways in relation to the fundamental questions about the solar five-minute oscillations: What excites them, what damps them, and why is their dominating period five minutes?

First of all, the numerical simulations confirm what has been surmised from the very beginning about the origin of the solar five-minute oscillations: the oscillations are stochastically excited by the turbulent convection, and they are damped by the collective response of the convection and radiation, with the main damping agent at low frequencies being the phase-shifted response of the turbulent pressure. This can be concluded both from the fact that oscillations are indeed stochastically excited in the numerical simulations (and are not overstable), to an amplitude that is comparable to the real solar oscillations. It is confirmed by the fact that the excitation power that can be directly measured in the simulations agrees to within the numerical scatter with the empirically determined excitation power, with a frequency dependence that is determined by the same asymptotic scaling relations that have been employed in the analytical estimates of the excitation power. In addition, the numerical simulations allow a detailed study of the physical sources of the excitation, with the possibility to study images of the horizontally averaged excitation (or proxys thereof) as a function of depth and time, or in full detail, as functions of three dimensions and time. The main cause of the excitation turns out to be the stochastic variations of the cooling rate at the surface, and the fluctuations of convective flux and turbulent pressure that are consequences thereof. 
The detailed mechanisms behind the linear damping of the modes are more difficult to separate, but the numerical results again confirm the picture that has been previously obtained by analytical methods (Gough, 1980; Balmforth, 1992a): namely that the main agent of damping at low frequencies is the phase-shifted, coherent response of the turbulent pressure to the oscillations. As pointed out by Balmforth, this effect cannot be described as simply a turbulent viscosity. On the contrary, it is strongly influenced by factors such as the coherent response of the buoyancy work to the oscillations, an effect that tends to force the response of the turbulent pressure in anti-phase with the density perturbations. There are additional terms in the differential equation that describes the time evolution of the turbulent pressure, and the combined effect of these terms is a phase lag that is such as to damp the coherent motions. The reason that the damping decreases more slowly with decreasing frequency than expected from naive estimates based on the near-surface amplitude of the evanescent modes is that the work integral picks up contributions from deeper layers at low frequencies. This is in agreement with the results of Gough (1980) and Balmforth (1992a).

The relatively more rapid decrease of the excitation power with decreasing frequency is in also in agreement with previous, analytic work (e.g., Balmforth, 1992c; Goldreich et al., 1994), and is caused by the more pronounced weighting of the excitation work integral towards the surface, relative to the work integral that controls the damping. As a result, the scaling of excitation power with frequency is primarily determined by the frequency behavior of the mode compressibility $\partial \xi_{\omega} / \partial r$, and peaks near the frequency where the logarithmic derivative $\partial \ln \left|\partial \xi_{\omega} / \partial r\right| / \partial \ln \omega$ is $\sim+2$, thus balancing the $\sim-2$ slope of the stochastic pressure fluctuations there. For the Sun, the result is that the power peaks at period of about 5 minutes. It is not obvious (at least from a superficial analysis) that the ratio of this frequency to the atmospheric cut-off frequency should be expected to be the same in stars whose surface structure differ substantially from that of the Sun.

Acknowledgments: The work of $\AA$.N was supported in part by the Danish Research Foundation, through its establishment of the Theoretical Astrophysics Center. RFS was supported by NASA grants NAG 1695 and NAG 5-4031 and NSF grant AST 9521785. The calculations were performed at the National Center for Supercomputer Applications, which is supported by the National Science Foundation, at Michigan State University and at UNI•C, Denmark. This valuable support is greatly appreciated.

\section{References}

Ando, H., Osaki, Y. 1977, PASJ, 29, 221

Antia, H. M., Chitre, S. M., Gough, D. O. 1987, in J. Christensen-Dalsgaard, S. Frandsen (eds.), Advances in Helio- and Asteroseismology, Vol. 123 of IAU Symp., 371

Antia, H. M., Chitre, S. M., Narasimha, D. 1982, Solar Phys., 77, 303

Bachmann, K. T., Schou, J., Brown, T. M. 1993, ApJ, 412, 870

Balmforth, N. J. 1992a, MNRAS, 255, 603

Balmforth, N. J. 1992b, MNRAS, 255, 632

Balmforth, N. J. 1992c, MNRAS, 255, 639

Biermann, L. 1946, Naturwiss., 33, 118

Biermann, L. 1948, Z. f. Astrophys., 25, 161

Christensen-Dalsgaard, J., Frandsen, S. 1983, Solar Physics, 82, 469

Deubner, F. L. 1975, A\&A, 44, 371

Dravins, D., Lindegren, L., Nordlund, A. 1981, A\&A, 96, 345 
Dravins, D., Lindegren, L., Nordlund, $\AA .1986$, A\&A, 158, 83

Dravins, D., Nordlund, A. 1990a, A\&A, 228, 184

Dravins, D., Nordlund, A. 1990b, A\&A, 228, 203

Evans, J. W., Michard, R. 1962, A\&A, 257, 287

Goldreich, P., Keeley, D. A. 1977, ApJ, 212, 243

Goldreich, P., Kumar, P. 1988, ApJ, 326, 462

Goldreich, P., Kumar, P. 1990, ApJ, 363, 694

Goldreich, P., Murray, N., Kumar, P. 1994, ApJ, 424, 466

Goode, P. R., Gough, D., Kosovichev, A. G. 1992, ApJ, 387, 707

Gough, D. O. 1980, in H. A. Hill, W. Dziembowski (eds.), Nonradial and Nonlinear Stellar Pulsations, Vol. 125 of Lecture Notes in Physics, 273

Kiselman, D., Nordlund, $\AA .1995$, A\&A, 302, 578

Leibacher, J., Stein, R. F. 1971, Ap. Letters, 7, 191

Leighton, R. B., Noyes, R. W., Simon, G. 1964, ApJ, 135, 474

Libbrecht, K. G. 1988, ApJ, 334, 510

Libbrecht, K. G., Woodard, M. F. 1991, Science, 253, 152

Lighthill, M. J. 1952, Proc. R. Soc. London, A211, 564

Moore, D. W., Spiegel, E. A. 1964, ApJ, 139, 48

Nordlund, А. 1982, A\&A, 107, 1

Nordlund, $\AA$. 1985, Solar Physics, 100, 209

Nordlund, $\AA$. 1991, in L. Crivellari, I. Hubeny, D. G. Hummer (eds.), Stellar Atmospheres: Beyond Classical Models, Kluwer

Nordlund, Å., Dravins, D. 1990, A\&A, 228, 155

Nordlund, A.., Spruit, H. C., Ludwig, H.-G., Trampedach, R. 1997, A\&A, 328, 229

Nordlund, A., Stein, R. F. 1990, Computer Phys. Communications, 59, 119

Nordlund, A., Stein, R. F. 1997, in F. P. Pijpers, J.Christensen-Dalsgaard, C. S. Rosenthal (eds.), Solar Convection, Oscillations and their Relationship; SCORe'96, Kluwer Academic Press, Dordrecht, (in press)

Nordlund, A., Stein, R. F. 1998, ApJ, (in preparation)

Osaki, Y. 1990, in Y. Osaki, H. Shibahashi (eds.), Progress of Seismology of the Sun and Stars, Springer-Verlag, Berlin, 145

Rast, M. P., Nordlund, §., Stein, R. F., Toomre, J. 1993, ApJ, 408, L53

Restaino, S. R., Stebbins, R. T., Goode, P. R. 1993, ApJ, 408, L57

Rimmele, T. R., Goode, P. R., Harold, E., Stebbins, R. T. 1995, ApJ, 444, L119

Rosenthal, C. S., Christensen-Dalsgaard, J., Nordlund, A., Stein, R. F., Trampedach, R. 1998, A\&A, (in preparation)

Schatzman, E. 1949, Ann. d'Astrophys, 12, 203

Schwarzschild, M. 1948, ApJ, 107, 1

Spruit, H. C., Nordlund, §., Title, A. 1990, ARA\&A, 28, 263

Stein, R. F. 1967, Solar Physics, 2, 385

Stein, R. F. 1968, ApJ, 297, 154

Stein, R. F., Nordlund, $\AA$. 1989, ApJ, 342, L95

Stein, R. F., Nordlund, $\AA$. 1991, in D. Gough, J. Toomre (eds.), Challenges to Theories of the Structure of Moderate Mass Stars, Vol. 388 of Lecture Notes in Physics, Springer, Heidelberg, p. 195

Stein, R. F., Nordlund, $\AA .1997$, ApJ, (in press)

Toutain, T., Frölich, C. 1992, ApJ, 412, 870

Ulrich, R. K. 1970, ApJ, 162, 993

Ulrich, R. K., Rhodes, E. J., J. 1977, ApJ, 218, 521

Unno, W. 1962, PASJ, 14, 416

Unno, W. 1964, Trans. Int. Astr. Un., XII(B), 555

Wöhl, H., Nordlund, Å. 1985, Solar Physics, 97, 213 